\title{
Impact of Psychiatric Diagnoses on Hospital Length of Stay in Children With Sickle Cell Anemia
}

\author{
Matthew P. Myrvik, PhD, ${ }^{1}$ Andrew D. Campbell, MD, ${ }^{1}$ Matthew M. Davis, MD, MAPP, ${ }^{1,2,3}$ and \\ Jennifer L. Butcher, PhD ${ }^{1}$
}

\begin{abstract}
Background. Patients with sickle cell anemia (SCA) experience a broad range of psychiatric disorders, placing them at risk for more complicated and longer hospitalizations for vaso-occlusive crises (VOC). The current study examined the frequency of psychiatric disorders in SCA patients (ages birth to 20 years) admitted for VOC in a nationally representative sample and the association between psychiatric disorders and hospital length of stay (LOS). Procedure. Patients with a primary diagnosis of SCA with crisis identified through the nationally representative Kids' Inpatient Database (KID) 2006 from the Agency of Healthcare Research and Quality were included for analysis. Patients with psychiatric disorders listed as secondary diagnoses were categorized under specific psychiatric disorders (mood disorder, anxiety disorder, disruptive behavior disorder, substance use disorder). Non-parametric and regression analyses were utilized for nationally weighted data,
\end{abstract}

to determine the effect of psychiatric disorders on LOS, while controlling for significant covariates. Results. For 21,255 hospital discharges for children with SCA with crisis in 2006, the mean LOS was 4.51 days. Approximately $6 \%$ of the patients discharged had a psychiatric disorder. After adjusting for significant covariates, mood disorders, anxiety disorders, and any psychiatric disorder were each associated with significantly longer LOS $(P<0.01)$. Substance and disruptive behavior disorders were not associated with LOS. Conclusions. Pediatric patients diagnosed with a psychiatric disorder, specifically mood or anxiety disorders, have longer LOS for VOC. These findings suggest that future interventions aimed at managing VOC may need to consider adjunctive psychiatric assessment and intervention. Pediatr Blood Cancer 2012;58:239-243. (c) 2011 Wiley Periodicals, Inc.

Key words: pain; psychology; sickle cell anemia

\section{INTRODUCTION}

Sickle cell anemia (SCA) is a genetic blood disorder affecting an estimated 90,000 persons, primarily African American, in the United States [1]. The clinical picture of SCA is characterized by recurrent vaso-occlusive crises (VOC) that result in pain and frequently require hospitalization for pain management. Within the pediatric population, VOC result in an estimated 19,000 hospitalizations per year in the United States and over 75,000 days spent in hospitals each year [2,3].

As a result of SCA and associated VOC, children and adolescents can experience negative consequences such as physical limitations, neurological complications, high rates of school absences, limited opportunities for socialization, and feelings of helplessness and self-consciousness [4,5]. Several investigators have reported that children and adolescents with SCA exhibit psychiatric symptoms. Bennett completed a meta-analysis investigating the rates of depression in youth with various medical conditions and concluded that children and adolescents with SCA have higher rates of depression than healthy peers [6]. Cepeda et al. [7] assessed psychiatric symptoms in a group of children with SCA through diagnostic interviews and found that $31 \%$ met diagnostic criteria for a psychiatric disorder. These authors also found frequency rates of $13 \%$ for mood disorders (major depression, dysthymia), 10\% for anxiety disorders (separation anxiety disorder, avoidant disorder, overanxious disorder), and $8 \%$ for disruptive behavior disorders (attention deficit/hyperactivity disorder, conduct disorder, oppositional defiant disorder).

Psychiatric diagnoses have been found to negatively impact the course of a number of pediatric chronic illnesses including pediatric rheumatic disorders and childhood diabetes [8,9]. Specific to SCA, studies examining adult and older adolescent patients have found an association between mental health symptoms and increased emergency department utilization and hospital admissions [10,11]. Furthermore, associations have been found between children's daily pain ratings and mood [12].
Previous studies suggest that psychiatric symptoms may place patients with SCA at a higher risk for difficulties managing the negative consequences of SCA, including pain. If true, then patients with psychiatric diagnoses may take longer to recover from VOC or have more complicated hospitalizations for VOC. To date, this association has not been examined. If psychiatric symptoms were found to independently predict increased LOS, these symptoms would represent a modifiable target to shorten LOS.

The objective of the current study was to determine the frequency of psychiatric diagnoses in a nationally representative sample of pediatric patients (ages birth to 20 years) diagnosed with SCA admitted for VOC. Additionally, the study aimed to determine the impact of psychiatric diagnoses on LOS in patients with SCA. It was hypothesized that patients diagnosed with SCA along with a psychiatric disorder would have a significantly longer LOS relative to patients diagnosed with SCA without a psychiatric disorder.

\section{METHODS}

\section{Participant Selection}

Data were obtained from the Healthcare Cost and Utilization Project (HCUP) Kids' Inpatient Database (KID) 2006, compiled

\footnotetext{
${ }^{1}$ Department of Pediatrics, University of Michigan, Ann Arbor, Michigan; ${ }^{2}$ Child Health Evaluation and Research (CHEAR) Unit, University of Michigan, Ann Arbor, Michigan; ${ }^{3}$ Gerald R. Ford School of Public Policy, University of Michigan, Ann Arbor, Michigan

Conflict of interest: Nothing to declare.

*Correspondence to: Matthew P. Myrvik, PhD, MRFC 3018, 8701 Watertown Plank Rd., Milwaukee, WI 53226.

E-mail: mmyrvik@mcw.edu
}

Received 1 November 2010; Accepted 9 February 2011 
by the Agency for Healthcare Research and Quality. This database provides national estimates of pediatric hospitalizations for childhood illnesses (birth to 20 years) through data collected from inpatient discharges from over 3,700 hospitals in 38 states. For this study, patients with a primary diagnosis (International Classification of Diseases, Ninth Revision, Clinical Modification; ICD-9-CM code 282.62) of SCA with crisis were selected from the KID for analysis. Children with ICD-9 codes for other sickle cell-related codes (e.g., SCA without crisis) as a primary diagnoses were excluded from analysis.

\section{Independent Variables}

Psychiatric disorders. Patients with any ICD-9-CM psychiatric diagnosis listed under secondary diagnoses were categorized as having a psychiatric disorder. Psychiatric disorder patients were further categorized into specific diagnosis subtypes: mood disorder, anxiety disorder, disruptive behavior disorder, and substance use disorder. Mood disorders were identified through diagnoses of any bipolar or depressive disorder (ICD-9-CM codes 296.x, 300.4, 309.0, 309.28, 311.0). Anxiety disorders were identified through diagnoses of any anxiety disorder (ICD-9-CM codes 300.00 , 300.01, 300.02, 300.2, 300.21, 300.23, 300.3, 309.24, 309.28, 309.81). Disruptive behavior disorders were identified through diagnoses of conduct disorder, oppositional defiant disorder, or attention deficit/hyperactivity disorder (ICD-9-CM codes 309.3, 312.8x, 313.81, 314.x). Substance use disorders were identified through diagnoses of alcohol or drug abuse or dependence, including tobacco (ICD-9-CM codes 291.x, 292.x, 303.x, 304.x, 305.x). Patients with multiple psychiatric diagnoses were coded into each specific diagnosis subtype, but were only coded once within the overall Psychiatric Disorder category.

\section{Dependent Variable}

Hospital length of stay (LOS). Hospital LOS was measured in days and calculated by subtracting the day of discharge from the day of admission. As such, same-day discharges were given the value of " 0. ."

\section{Confounding Variables}

Patient demographics, hospital characteristics, and secondary diagnoses and medical procedures associated with increased complication during hospitalization were examined as potential confounding variables. Variables were chosen that had been demonstrated by previous research to have an independent effect on LOS in SCA patients [2,3,5].

Patient demographics. Patient gender, age, and primary payer for hospital services were examined as potential confounding variables. Age was calculated based on date of birth relative to the time of admission and divided into four categories across childhood: Birth to 5 years (early childhood), 6-11 years (school age), 12-17 years (adolescence), and 18-20 years (young adult). Primary payer for hospital services was coded into six categories: Medicare, Medicaid, private insurance, self-pay, no charges, and other payer.

Hospital characteristics. Hospital location/teaching status was categorized by the KID into rural, urban teaching, and urban non-teaching. Teaching status was determined using information Pediatr Blood Cancer DOI 10.1002/pbc from the American Hospital Association (AHA) Annual Survey Database. Hospital region was divided into four areas: Northeast (CT, MA, NH, NJ, NY, RI, VT), Midwest (IL, IN, IA, KS, MI, $\mathrm{MN}, \mathrm{MO}, \mathrm{NE}, \mathrm{OH}, \mathrm{SD}, \mathrm{WI})$, West (AZ, CA, CO, HI, NV, OR, UT, WA), and South (AR, DC, FL, GA, KY, MD, NC, OK, SC, TN, TX, VA, WV).

Secondary diagnoses and medical procedures. Secondary diagnoses of pneumonia, asthma, and constipation and the total number of secondary diagnoses were examined as potential confounding variables. The number of secondary diagnoses was categorized into three groups: $1,2-4$, and 5 or more diagnoses. Additionally, the medical procedure of blood transfusion was examined as a potential confounding variable, given its likely utility as a marker of severity of illness during the hospitalization.

\section{Statistical Analyses}

All child and hospital variables were examined using a weighted KID sample, with weights provided within the KID to permit inferences regarding national patterns of illness and care. Analysis of LOS revealed that this variable was positively skewed, as is typical for LOS data. As such, LOS is presented as unadjusted LOS data and adjusted LOS data using log transformation. A Mann-Whitney $U$-test was performed to examine differences in LOS by psychiatric diagnosis category. Linear regression analysis was performed to examine the effect of psychiatric diagnoses on adjusted LOS while controlling for significant covariates. The regression model provides an estimate of the $\log$ of the probability that a hospital stay will end on a certain day. Variables entered into the regression model were those found to be significantly related through independent bivariate regressions. Parameter estimates were performed and results are presented as estimates of the relative difference between groups.

\section{RESULTS}

Patient demographics are shown in Table I. In 2006, there were an estimated 21,255 hospital discharges for children and adolescents with a primary diagnosis of SCA with crisis. The mean age at the time of admission was 13.0 years $( \pm 5.93$ years, range $0-20$ years) and $50.4 \%$ of the patients discharged were female. The mean unadjusted LOS was 4.51 days $( \pm 4.28$ days, range 0 108 days).

Records with missing data were excluded from analysis and accounted for less than $1 \%$ of the data. Based on independent bivariate regressions, the final regression model included: age; hospital region; hospital location/teaching status; primary payer; number of secondary diagnoses; secondary diagnoses of pneumonia, asthma, and constipation; and a blood transfusion. Similar to previous studies, gender was not found to be significantly related to LOS and was not included in the final regression model $[2,3]$.

\section{Psychiatric Disorders in SCA}

In 2006, approximately $6 \%$ of the patients discharged with a primary diagnosis of SCA with crisis had a psychiatric diagnosis. Mood disorders and substance use disorders accounted for the majority of the psychiatric discharge diagnoses. Of the patients with SCD and a psychiatric diagnosis, $63 \%$ were ages 1820 years, 32\% were ages $12-17$ years, 5\% were 6-11 years, 
TABLE I. Psychiatric Disorders and Patient Demographics Included in the Regression Model

\begin{tabular}{|c|c|}
\hline Characteristics & $\mathrm{N}(\%)$ \\
\hline Psychiatric disorder & $1,318(6)$ \\
\hline Mood disorder & $562(2)$ \\
\hline Depressive disorder NOS & $495(2)$ \\
\hline Anxiety disorder & $192(<1)$ \\
\hline Anxiety disorder NOS & $107(<1)$ \\
\hline Substance disorder & $545(2)$ \\
\hline Nicotine dependence & $369(1)$ \\
\hline Disruptive behavior disorder & $156(<1)$ \\
\hline ADHD (combined type) & $103(<1)$ \\
\hline \multicolumn{2}{|l|}{ Patient demographics } \\
\hline \multicolumn{2}{|l|}{ Age } \\
\hline Birth-5 years & $3,449(16)$ \\
\hline $6-11$ years & $4,213(19)$ \\
\hline $12-17$ years & $7,577(35)$ \\
\hline $18-20$ years & $6,348(29)$ \\
\hline \multicolumn{2}{|l|}{ Primary payer } \\
\hline Medicare & $204(<1)$ \\
\hline Medicaid & $14,230(66)$ \\
\hline Private insurance & $5,543(26)$ \\
\hline Self-pay & $792(4)$ \\
\hline No charge & $48(<1)$ \\
\hline Other payer & $827(4)$ \\
\hline \multicolumn{2}{|l|}{ Secondary diagnoses } \\
\hline Pneumonia & $2,426(11)$ \\
\hline Asthma & $3,257(15)$ \\
\hline Constipation & $2,012(9)$ \\
\hline \multicolumn{2}{|l|}{ Number of diagnoses } \\
\hline 1 & $4,583(21)$ \\
\hline $2-4$ & $12,492(58)$ \\
\hline $5+$ & $4,569(21)$ \\
\hline \multicolumn{2}{|l|}{ Procedure } \\
\hline Blood transfusion & $5,402(25)$ \\
\hline
\end{tabular}

and none were ages $0-5$ years. Mood disorders, anxiety disorders, and substance use disorders were more frequent in patients ages 18-20 years; whereas disruptive behavior disorders were more frequent in patients ages $12-17$ years. Approximately $6.9 \%$ of patients with public insurance had a concomitant psychiatric diagnosis and $4.3 \%$ of patients with private insurance had a concomitant psychiatric diagnosis. Frequencies of the psychiatric disorder categories and most prominent individual discharge psychiatric diagnoses are included in Table I.

\section{LOS and Psychiatric Diagnoses}

In unadjusted analyses, LOS was found to be significantly longer in patients with a psychiatric disorder $(P<0.001)$ relative to patients without a psychiatric disorder. Mean unadjusted LOS was 4.4 days for patients without a psychiatric disorder and increased by $2.3-6.7$ days in patients with a psychiatric disorder (Table II). This difference persisted after adjusting for confounding variables $(P=0.007)$. While controlling for significant covariates, LOS for patients with SCA and a psychiatric diagnosis was $7 \%$ longer than for patients without a psychiatric disorder (Table III).
TABLE II. Unadjusted Length of Stay (LOS) for Psychiatric Diagnoses

\begin{tabular}{lccc}
\hline Category & Diagnosis $^{\mathrm{a}}$ & No diagnosis $^{\mathrm{a}}$ & \multicolumn{1}{c}{$P$} \\
\hline Psychiatric disorder & $6.7(6.14-7.16)$ & $4.4(4.29-4.44)$ & $<0.001$ \\
Mood disorder & $7.4(6.68-8.21)$ & $4.4(4.36-4.51)$ & $<0.001$ \\
Anxiety disorder & $8.4(7.10-9.77)$ & $4.5(4.40-4.55)$ & $<0.001$ \\
Disruptive behavior & $5.4(4.16-6.65)$ & $4.5(4.43-4.58)$ & 0.001 \\
$\quad$ disorder & & & \\
Substance disorder & $5.7(4.94-6.55)$ & $4.5(4.40-4.55)$ & 0.344 \\
\hline${ }^{a}$ Unadjusted mean LOS in days (95\%CI). & \\
\end{tabular}

\section{LOS and Psychiatric Diagnosis Subtypes}

In unadjusted analyses, LOS was significantly longer in patients with SCA and mood disorders $(P<0.001)$, anxiety disorders $(P<0.001)$, and substance disorders $(P=0.001)$ relative to patients without psychiatric disorders. Unadjusted LOS increased by 3.0 days for patients with mood disorders, 3.9 days for patients with anxiety disorders, and 1.2 days for patients with substance disorders relative to patients without a psychiatric disorder (Table II). This relationship persisted for mood disorders $(P<0.001)$ and anxiety disorders $(P<0.001)$ after controlling for confounding variables independently related to LOS. Adjusted LOS was found to be $17 \%$ longer for patients with mood disorders and 23\% longer for patients with anxiety disorders relative to patients with no psychiatric disorder. Unexpectedly, patients with substance use disorders were found to have a shorter LOS in adjusted analyses $(P=0.01)$. Unadjusted LOS was not significantly increased in patients with a comorbid disruptive behavior disorder, in either unadjusted or adjusted analyses.

TABLE III. Linear Regression Analysis of Effect of Psychiatric Diagnosis on Adjusted Hospital LOS

\begin{tabular}{|c|c|c|c|}
\hline Category & $\begin{array}{l}\text { Parameter } \\
\text { estimate }^{\mathrm{a}}\end{array}$ & $95 \% \mathrm{CI}$ & $P$ \\
\hline \multicolumn{4}{|c|}{ Psychiatric disorder } \\
\hline Diagnosis & 1.07 & $1.02-1.12^{\mathrm{b}}$ & \multirow[t]{2}{*}{0.007} \\
\hline No diagnosis & 1.0 & Referent & \\
\hline \multicolumn{4}{|l|}{ Mood disorder } \\
\hline Diagnosis & 1.17 & $1.09-1.24^{\mathrm{b}}$ & \multirow[t]{2}{*}{$<0.001$} \\
\hline No diagnosis & 1.0 & Referent & \\
\hline \multicolumn{4}{|l|}{ Anxiety disorder } \\
\hline Diagnosis & 1.23 & $1.12-1.34^{\mathrm{b}}$ & \multirow[t]{2}{*}{$<0.001$} \\
\hline No diagnosis & 1.0 & Referent & \\
\hline \multicolumn{4}{|c|}{ Disruptive behavior disorder } \\
\hline Diagnosis & 0.93 & $0.81-1.04$ & \multirow[t]{2}{*}{0.165} \\
\hline No diagnosis & 1.0 & Referent & \\
\hline \multicolumn{4}{|c|}{ Substance disorder } \\
\hline Diagnosis & 0.90 & $0.83-0.97^{\mathrm{b}}$ & \multirow[t]{2}{*}{0.01} \\
\hline No diagnosis & 1.0 & Referent & \\
\hline
\end{tabular}

${ }^{\mathrm{a}}$ Interpreted as \% increased stay; ${ }^{\mathrm{b}}$ Variable statistically significant within linear regression model. Other significant covariates are age, primary payer, number of diagnoses, pneumonia, asthma, constipation, blood transfusion, hospital region, and hospital location/ teaching status. 


\section{DISCUSSION}

SCA is characterized by recurrent VOC that frequently require hospitalization for pain management. Previous research has found that patients with SCA are at risk for negative life consequences likely related to the pain associated with this chronic illness, which may place a patient at risk for increased negative consequences associated with SCA. Our study describes the frequency of psychiatric diagnoses in a nationally representative sample and to examine the association between psychiatric diagnoses and LOS for VOC in pediatric patients with SCA.

Overall, psychiatric diagnoses were found in approximately $6 \%$ of the SCA sample, with mood disorders and substance use disorders being the most frequent disorders. Additionally, after controlling for confounding variables (i.e., patient demographics and hospital characteristics, secondary diagnoses, and blood transfusions), pediatric patients with SCA and a psychiatric diagnosis were found to have significantly longer LOS relative to patients without a psychiatric diagnosis. In particular, patients with psychiatric diagnoses remained hospitalized more than 2 days longer than patients without a psychiatric diagnosis.

When examining individual psychiatric diagnoses, results were somewhat inconsistent. Patients diagnosed with mood disorders or anxiety disorders remained hospitalized 3 or more days longer. However, substance use disorders and disruptive behavior disorders were not associated with significantly longer LOS. Unexpectedly, substance use disorders were associated with a shorter LOS.

These results generally support previous research findings highlighting the negative association between psychiatric diagnoses and SCA pain $[13,14]$ and suggest that psychiatric diagnoses may negatively impact adaptive management of VOC. There may be multiple reasons for the findings associated with mood and anxiety disorders. First, symptoms associated with depression or anxiety can resemble pain-related symptoms. Patients with these disorders may experience sleep disturbance, appetite disturbance, fatigue, or impaired physical functioning, which are similar to symptoms experienced during a VOC. Thus, patients may remain hospitalized longer due to mood-related symptoms exacerbating or mimicking pain-related symptoms. Second, a patient's mental illness may impact his or her ability to manage a VOC episode. It has been shown that certain coping strategies (i.e., passive coping, negative thought patterns) associated with mood and anxiety disorders may impact a patient's ability to manage pain in SCA $[13,15,16]$.

Unexpectedly, substance use and disruptive behavior disorders were not associated with LOS. However, the primary substance use disorder was nicotine dependence, which may actually facilitate a patient's ability to manage pain as experimental studies suggest that nicotine has analgesic properties [17-19]. Additionally, the primary disruptive behavior disorder was attention deficit/hyperactivity disorder. As ADHD is often associated with younger children and age has been independently related to LOS [3], controlling for age may have removed the specific variance associated with disruptive behavior disorders.

There are a number of limitations of this study to consider. First, this is a cross-sectional study, and caution should be taken when drawing conclusions over time; causality cannot be ascertained with certainty. For example, psychiatric disorders may impact a patient's ability to manage their pain resulting in a longer hospital admission. However, patients demonstrating longer hospital admissions may be more likely to be diagnosed with a psychiatric disorder as medical providers may order additional consultations to provide information for a patient's prolonged hospital admission. Second, the data did not identify individuals with frequent readmissions, who may influence the data. Finally, psychiatric disorders were identified via discharge diagnoses using ICD-9-CM codes and not formal psychiatric screening. The current coding strategy allowed for convenience, but prevented a specific understanding of how the diagnosis was made. Studies utilizing clinical interviews and standardized parent- or teacher-report questionnaires have found that the incidence of comorbid psychiatric diagnosis in children with SCA ranges from $22 \%$ to $50 \%$; whereas the current study utilized existing ICD-9CM codes and found that $6 \%$ of the sample had a comorbid psychiatric diagnosis [7,20-22]. Overall, the current coding strategy may under-represent the incidence of psychiatric comorbidities within children with SCA.

In summary, patients with a psychiatric diagnosis, more specifically mood and anxiety disorders, and SCA had a longer LOS than patients without a psychiatric diagnosis. This relationship supports the hypothesis that psychiatric symptoms impact LOS in patients hospitalized for VOC. Future research aimed at interventions to manage VOC should consider adjunctive psychiatric treatments. Empirical evidence is growing and suggesting that psychiatric interventions, such as cognitive behavioral therapy, can effectively aid patients with pain management, and coping with SCA [23]. Through psychiatric assessment and intervention, patients with SCA may learn to more effectively cope with pain, which could subsequently reduce hospital LOS.

\section{ACKNOWLEDGMENT}

The authors acknowledge James Gurney, Ph.D. (Department of Pediatrics) and Joe Kazemi, M.A. (Center for Statistical Consultation and Research) of the University of Michigan for statistical support. The authors also acknowledge Julie Panepinto, M.D., M.S.P.H. and Andrea Morrison, M.D. (Department of Pediatrics) of the Medical College of Wisconsin for manuscript review.

\section{REFERENCES}

1. Brousseau DC, Panepinto JA, Nimmer M, et al. The number of people with sickle-cell disease in the United States: National and state estimates. Am J Hematol 2010;85:77-78.

2. Ellison AM, Bauchner H. Socioeconomic status and length of hospital stay in children with vasoocclusive crises of sickle cell disease. J Natl Med Assoc 2007;99:192-196.

3. Panepinto JA, Brousseau DC, Hillery CA, et al. Variation in hospitalizations and hospital length of stay in children with vaso-occlusive crises in sickle cell disease. Pediatr Blood Cancer 2005;44:182-186.

4. Brown RT, Kaslow NJ, Doepke K, et al. Psychosocial and family functioning in children with sickle cell syndrome and their mothers. J Am Acad Child Adolesc Psychiatry 1993;32:545-553.

5. Merenstein D, Egleston B, Diener-West M. Lengths of stay and costs associated with children's hospitals. Pediatrics 2005;115:839-844.

6. Bennett DS. Depression among children with chronic medical problems: A meta-analysis. J Pediatr Psychol 1994;19:149-169.

7. Cepeda ML, Yang YM, Price CC, et al. Mental disorders in children and adolescents with sickle cell disease. South Med J 1997;90:284-287.

8. Brand AH, Johnson JH, Johnson SB. Life stress and diabetic control in children and adolescents with insulin-dependent diabetes. J Pediatr Psychol 1986;11:481-495.

9. von Weiss RT, Rapoff MA, Varni JW, et al. Daily hassles and social support as predictors of adjustment in children with pediatric rheumatic disease. J Pediatr Psychol 2002;27:155-165.

10. Belgrave FZ, Molock SD. The role of depression in hospital admissions and emergency treatment of patients with sickle cell disease. J Natl Med Assoc 1991;83:777-781.

11. Hasan SP, Hashmi S, Alhassen M, et al. Depression in sickle cell disease. J Natl Med Assoc 2003;95: 533-537.

12. Gil KM, Carson JW, Porter LS, et al. Daily stress and mood and their association with pain, health-care use, and school activity in adolescents with sickle cell disease. J Pediatr Psychol 2003;28:363-373.

13. Barakat LP, Schwartz LA, Simon K, et al. Negative thinking as a coping strategy mediator of pain and internalizing symptoms in adolescents with. J Behav Med 2007;30:199-208. 
14. Wison Schaeffer JJ, Gil KM, Burchinal M, et al. Depression, disease severity, and sickle cell disease. J Behav Med 1999;22:115-126.

15. Gil KM, Abrams MR, Phillips G, et al. Sickle cell disease pain: Relation of coping strategies to adjustment. J Consult Clin Psychol 1989;57:725-731.

16. Gil KM, Edens JL, Wilson JJ, et al. Coping strategies and laboratory pain in children with sickle cell

disease. Ann Behav Med 1997;19:22-29.
17. Aceto MD, Awaya H, Martin BR, et al. Antinociceptive action of nicotine and its methiodide derivatives in mice and rates. Br J Pharmacol 1983;79:869-876.

18. Jamner LD, Girdler SS, Shapiro D, et al. Pain inhibition, nicotine, and gender. Exp Clin Psychopharmacol 1998;6:96-106.
19. Pomerleau OF, Turk DC, Fertig JB. The effects of cigarette smoking on pain and anxiety. Addict Behav 1984;9:265-281.

0. Benton TD, Ifeagwu JA, Smith-Whitley K. Anxiety and depression in children and adolescents with sickle cell disease. Curr Psychiatry Rep 2007;9:114-121.

21. Iloeje SO. Psychiatric morbidity among children with sickle-cell disease. Dev Med Child Neurol 1991;33:1087-1094

22. Thompson RJ Jr, Gil KM, Burbach DJ, et al. Role of child and maternal processes in the psychological adjustment of children with sickle cell disease. J Consult Clin Psychol 1993;61:468-474.

23. Chen E, Cole SW, Kato PM. A review of empirically supported psychosocial interventions for pain and adherence outcomes in sickle cell disease. J Pediatr Psychol 2004;29:197-209. 